\title{
Clinical characteristics and outcomes of null-cell versus silent gonadotroph adenomas in a series of 1166 pituitary adenomas from a single institution
}

\author{
Alexander F. Haddad, BS, ${ }^{1}$ Jacob S. Young, MD, ${ }^{3}$ Taemin Oh, MD, ${ }^{3}$ Matheus P. Pereira, BS, ${ }^{1}$ \\ Rushikesh S. Joshi, BS, ${ }^{2}$ Kaitlyn M. Pereira, BS, ${ }^{4}$ Robert C. Osorio, BS, ${ }^{1}$ Kevin C. Donohue, BS, ${ }^{1}$ \\ Zain Peeran, BS, ${ }^{3}$ Sweta Sudhir, BS, ${ }^{3}$ Saket Jain, PhD, ${ }^{3}$ Angad Beniwal, BS, ${ }^{3}$ \\ Ashley S. Chopra, BA, ${ }^{3}$ Narpal S. Sandhu, BA, ${ }^{3}$ Philip V. Theodosopoulos, MD, ${ }^{3}$ \\ Sandeep Kunwar, MD, ${ }^{3}$ Ivan H. El-Sayed, MD, ${ }^{5}$ José Gurrola II, MD, ${ }^{5}$ Lewis S. Blevins Jr., MD, ${ }^{3}$ \\ and Manish K. Aghi, MD, PhD ${ }^{3}$
}

${ }^{1}$ School of Medicine, University of California, San Francisco; ${ }^{2}$ School of Medicine, University of California, San Diego; ${ }^{3}$ Department of Neurological Surgery, University of California, San Francisco, California; ${ }^{4}$ University of South Florida Morsani College of Medicine, Tampa, Florida; and ${ }^{5}$ Department of Otolaryngology-Head and Neck Surgery, University of California, San Francisco, California

OBJECTIVE Nonfunctioning pituitary adenomas present without biochemical or clinical signs of hormone excess and are the second most common type of pituitary adenomas. The $2017 \mathrm{WHO}$ classification scheme of pituitary adenomas differentiates null-cell adenomas (NCAs) and silent gonadotroph adenomas (SGAs). The present study sought to highlight the differences in patient characteristics and clinical outcomes between NCAs and SGAs.

METHODS The records of 1166 patients who underwent transsphenoidal surgery for pituitary adenoma between 2012 and 2019 at a single institution were retrospectively reviewed. Patient demographics and clinical outcomes were collected.

RESULTS Of the overall pituitary adenoma cohort, 12.8\% $(n=149)$ were SGAs and 9.2\% $(n=107)$ NCAs. NCAs were significantly more common in female patients than SGAs $(61.7 \%$ vs $26.8 \%, p<0.001)$. There were no differences in patient demographics, initial tumor size, or perioperative and short-term clinical outcomes. There was no significant difference in the amount of follow-up between patients with NCAs and those with SGAs (33.8 months vs 29.1 months, $p=$ $0.237)$. Patients with NCAs had significantly higher recurrence $(p=0.021)$, adjuvant radiation therapy usage $(p=0.002)$, and postoperative diabetes insipidus $(p=0.028)$. NCA pathology was independently associated with tumor recurrence (HR 3.64, 95\% Cl 1.07-12.30; $p=0.038$ ), as were cavernous sinus invasion (HR 3.97, 95\% Cl 1.04-15.14; $p=0.043$ ) and anteroposterior dimension of the tumor ( $\mathrm{HR} 2.23,95 \% \mathrm{Cl} 1.09-4.59 ; \mathrm{p}=0.030)$.

CONCLUSIONS This study supports the definition of NCAs and SGAs as separate subgroups of nonfunctioning pituitary adenomas, and it highlights significant differences in long-term clinical outcomes, including tumor recurrence and the associated need for adjuvant radiation therapy, as well as postoperative diabetes insipidus. The authors also provide insight into independent risk factors for these outcomes in the adenoma population studied, providing clinicians with additional predictors of patient outcomes. Follow-up studies will hopefully uncover mechanisms of biological aggressiveness in NCAs and associated molecular targets.

https://thejns.org/doi/abs/10.3171/2020.3.FOCUS20114

KEYWORDS pituitary adenoma; null-cell adenoma; silent gonadotroph adenoma

ABBREVIATIONS AP = anteroposterior; GTR = gross-total resection; ML = medial-lateral; NCA = null-cell adenoma; NFPA = nonfunctioning pituitary adenoma; SGA = silent gonadotroph adenoma; SI = superior-inferior; SPA = silent pituitary adenoma; STR = subtotal resection; TVDT = tumor volume doubling time; UCSF = University of California, San Francisco.

SUBMITTED February 3, 2020. ACCEPTED March 4, 2020.

INCLUDE WHEN CITING DOI: 10.3171/2020.3.FOCUS20114. 
$\mathrm{P}$ ITUITARY adenomas account for $10 \%-20 \%$ of all intracranial tumors, with an increasing incidence in the United States., ${ }^{4,6}$ A large number of adenomas are clinically nonfunctioning pituitary adenomas (NFPAs) without clinical or laboratory evidence of excess hormone secretion, but with symptoms related to mass effect of the tumor, including headaches and visual field defects, that frequently prompt their discovery. ${ }^{5,17}$ NFPAs are considered to be the most common type of adenoma after prolactinomas, and are thought to represent $14 \%-54 \%$ of all pituitary adenomas, although estimates vary depending on the study., $4,17,21$

Pathological analysis of NFPA tissue enables NFPAs to be divided into silent pituitary adenomas (SPAs) versus null-cell adenomas (NCAs), a distinction that was formalized in 2017 when the WHO criteria were modified to include these entities. SPAs are adenomas with evidence of hormone or transcription factor staining on final pathology results, but otherwise no clinical or biochemical evidence of significant hormone excess. ${ }^{6}, 16$ Thus, 90\%-99\% of NFPAs are found to be SPAs following final pathology results, frequently depending on the number of pathological stains performed ${ }^{6,16}$ All types of adenomas can be silent, including somatotroph-, lactotroph-, thyrotroph-, corticotroph-, gonadotroph-, and PIT-1-positive adenomas, with biochemical expression of hormones seen occurring on a spectrum rather than in a binary manner. ${ }^{1,6,13,14}$ NCAs are NFPAs that on final pathology findings do not stain positive for any pituitary hormones or transcription factors. ${ }^{6,14}$ In 2015, Nishioka et al. demonstrated the importance of including the transcription factor staining in addition to hormone staining in the differentiation of NCAs and SPA subtypes. ${ }^{16}$ Indeed, this was especially true for silent gonadotroph adenomas (SGAs), with 79/119 hormone-negative adenomas staining positive for SF-1, a marker of gonadotroph lineage. This study contributed to the 2017 WHO classification scheme differentiating between SF-1-positive SPAs and NCAs, although comprehensive transcription factor staining is still not available in many pathology laboratories. ${ }^{14}$

Classification of the SPA subtype is critical given the potential clinical ramifications for patients. Studies have highlighted its importance by showing that silent corticotroph adenomas, for example, tend to present with increased cavernous sinus invasion and when patients are younger. ${ }^{11,16}$ Across all subtypes, SPAs are more aggressive relative to their functional adenoma counterparts. ${ }^{6}$ Given that many of the tumors previously considered to be NCAs are in fact SF-1-positive SGAs, it is vital to consider the differences between these 2 groups. ${ }^{2,6}$ In their cohort of 31 NCAs and 63 SGAs, Balogun et al. detailed differences in tumor behavior between the 2 subtypes, demonstrating higher MIB-1 staining and a positive correlation between pre- and postoperative tumor volume doubling times (TVDTs) in NCAs. ${ }^{2}$ Their study was limited to behavioral characteristics of the 2 adenoma types and did not assess clinical outcomes aside from resection rates. ${ }^{12}$ There remains a severe paucity of data investigating the difference between NCAs and SGAs with regard to clinical outcomes.

Our study sought to describe how patient character- istics as well as short- and long-term clinical outcomes, including the need for adjuvant radiation therapy and incidence of diabetes insipidus, differ between SGAs and NCAs. We leveraged a database of 1166 patients from a single institution with pituitary adenomas and extensive follow-up periods spanning 2012 to 2019 to assess any potential differences between the groups.

\section{Methods \\ Patient Population}

A retrospective review of the University of California, San Francisco (UCSF) Department of Neurosurgery pituitary database was done in order to identify patients who had undergone transsphenoidal surgery for a pituitary adenoma by the senior author (M.K.A.) and practice partners (S.K. and P.V.T.) between May 2012 and October 2019. This study was approved by the UCSF institutional review board. Patients with SGAs were defined as having an NFPA, per clinical records, with pathological specimens staining positive for SF-1 and negative for adrenocorticotropic hormone, prolactin, and growth hormone. SGAs were also either negative or untested for thyroid-stimulating hormone, PIT-1, and TPIT, contingent on whether testing was available during the year of surgery. NCAs were defined as clinically nonfunctioning pituitary adenomas negative for SF-1, adrenocorticotropic hormone, prolactin, and growth hormone. NCAs were also either negative or untested for thyroid-stimulating hormone, PIT-1, and TPIT, contingent on whether testing was available during the year of surgery. The decision to recommend radiosurgery or fractionated radiation therapy is made by the treatment team at our institution, including neurosurgeons, endocrinologists, and radiation oncologists. NFPAs, such as the adenomas included in this study, receive radiation for residual tumor noted to be present on a 6-month followup MRI, with radiosurgery used for small residual lesions in the cavernous sinus and fractionated radiation therapy used for larger residual lesions extending into the intracranial compartment. NFPAs also receive radiation for small tumor recurrence noted on serial imaging that is in the cavernous sinus and thus amenable to radiosurgery.

\section{Imaging Analysis}

The radiologist's measurements were used for all adenomas when noted in the medical records. A single research associate performed all measurements for tumors that lacked radiologist-defined measurements. Macroadenomas were defined as having any dimension with a length greater than or equal to $10 \mathrm{~mm}$. Microadenomas were defined as adenomas with all tumor dimensions less than $10 \mathrm{~mm}$. Gross-total resection (GTR) versus subtotal resection (STR) was determined by a radiologist using postoperative MRI scans. Cavernous sinus invasion was determined by a radiologist using preoperative MRI scans.

\section{Statistical Analysis}

Chi-square and Student t-tests were used to compare categorical and continuous variables, respectively, between the groups. A backward likelihood ratio Cox re- 
TABLE 1. Demographic data in 1166 patients with pituitary adenomas

\begin{tabular}{|c|c|c|c|}
\hline Variable & NCA & SGA & $\mathrm{p}$ Value \\
\hline No. of patients & $107(9.2 \%)$ & $149(12.8 \%)$ & \\
\hline Age at surgery, yrs (range) & $58.1(18-93)$ & $59.0(26-92)$ & 0.892 \\
\hline Female sex & $66(61.7 \%)$ & $40(26.8 \%)$ & $<0.001$ \\
\hline \multicolumn{4}{|c|}{ Radiographic parameters, cm (range) } \\
\hline $\mathrm{AP}$ & $1.99(0.1-6.2)$ & $2.15(0.2-6.5)$ & 0.198 \\
\hline $\mathrm{ML}$ & $2.04(0.30-6.60)$ & $2.16(0.10-4.2)$ & 0.640 \\
\hline SI & $2.09(0.40-6.60)$ & $2.31(0.10-5.10)$ & 0.492 \\
\hline Diameter & $2.06(0.33-6.47)$ & $2.21(0.13-4.80)$ & 0.463 \\
\hline Adenoma size & & & 0.404 \\
\hline Microadenoma & $4(3.7 \%)$ & $3(2.0 \%)$ & \\
\hline Macroadenoma & $103(96.3 \%)$ & $146(98.0 \%)$ & \\
\hline No. of prior pituitary surgeries & & & 0.635 \\
\hline 0 & $91(85.0 \%)$ & $120(80.5 \%)$ & \\
\hline 1 & $12(11.2 \%)$ & $25(16.8 \%)$ & \\
\hline 2 & $3(2.8 \%)$ & $3(2.0 \%)$ & \\
\hline 3 & $1(0.9 \%)$ & $1(0.7 \%)$ & \\
\hline 4 & $0(0.0 \%)$ & $0(0.0 \%)$ & \\
\hline Type of procedure & & & 0.404 \\
\hline Endoscopic & $34(31.8 \%)$ & $39(26.2 \%)$ & \\
\hline Microscopic & $70(65.4 \%)$ & $108(72.5 \%)$ & \\
\hline Both & $3(2.8 \%)$ & $2(1.3 \%)$ & \\
\hline MIB-1 index (range)* & $2.16(0-9)$ & $1.70(1-5)$ & 0.422 \\
\hline p53 immunopositivity† & $17(53.1 \%)$ & $44(72.1 \%)$ & 0.041 \\
\hline Cavernous sinus invasion & $46(43.0 \%)$ & $55(36.9 \%)$ & 0.326 \\
\hline Sphenoid sinus invasion & $16(15.0 \%)$ & $20(13.4 \%)$ & 0.728 \\
\hline Any neoadjuvant radiation & $5(4.7 \%)$ & $6(4.0 \%)$ & 0.555 \\
\hline Radiosurgery & $1(0.9 \%)$ & $1(0.7 \%)$ & 0.813 \\
\hline Radiotherapy & $4(3.7 \%)$ & $5(3.4 \%)$ & 0.870 \\
\hline Preexisting diabetes insipidus & $0(0.0 \%)$ & $2(1.3 \%)$ & 0.229 \\
\hline Follow-up length, mos (range) & $33.82(0-89)$ & $29.12(0-84)$ & 0.237 \\
\hline
\end{tabular}

gression model was used to assess variables independently associated with recurrence in patients with either an SGA or NCA. Starting variables for the model included the following: patient sex; age at surgery; anteroposterior (AP) measurement; medial-lateral (ML) measurement; superior-inferior (SI) measurement; tumor diameter; macroadenoma versus microadenoma; extent of resection; neoadjuvant radiation; tumor cavernous sinus invasion; tumor sphenoid sinus invasion; and SGA versus NCA pathology. Starting variables for the binary logistic model assessing new diabetes insipidus included the following: patient sex; age at surgery; AP measurement; ML measurement; SI measurement; macroadenoma versus microadenoma; extent of resection; neoadjuvant radiation; intraoperative CSF encounter; postoperative CSF leak; hyponatremia; tumor cavernous sinus invasion; tumor sphenoid sinus invasion; and SGA versus NCA pathology. Statistical significance was defined as a $p$ value less than 0.05 . All analysis was performed using SPSS 26 (IBM Corp.).

\section{Results}

\section{Patient Clinical Demographic Data}

We identified 1166 patients with pituitary adenomas as having had transsphenoidal surgery at our center between 2012 and 2019, of whom 107 (9.2\%) patients with an NCA and $149(12.8 \%)$ with an SGA were identified. Patient characteristics within the subgroups are listed in Table 1. There was no significant difference in patient age between the group of patients with NCAs and the group with SGAs (58.1 years and 59 years, $\mathrm{p}=0.892)$. SGAs occurred in a significantly lower number of female patients than did NCAs $(26.8 \%$ vs $61.7 \%, p<0.001)$. There were no significant differences in tumor size in any dimension or macroadenoma/microadenoma designation between NCAs and SGAs. There was no significant difference in the number of prior surgeries between the 2 groups (Table 1). MIB-1 staining, neoadjuvant radiation, and incidence of preexisting diabetes insipidus were also not significant- 
TABLE 2. Perioperative complications and short-term outcomes in 1166 patients with pituitary adenomas

\begin{tabular}{lccc}
\hline \multicolumn{1}{c}{ Variable* $^{*}$} & NCA & SGA & p Value \\
\hline Length of stay (range) & $2.82(1-14)$ & $2.44(1-31)$ & 0.506 \\
\hline Drain & & & 0.072 \\
\hline None & $91(85.0 \%)$ & $139(93.3 \%)$ & \\
\hline Lumbar drain & $15(14.0 \%)$ & $10(6.7 \%)$ & \\
\hline EVD & $1(0.9 \%)$ & $0(0.0 \%)$ & \\
\hline Intraop CSF encounter & $27(25.2 \%)$ & $28(18.8 \%)$ & 0.216 \\
\hline Periop complication & $29(27.1 \%)$ & $32(21.5 \%)$ & 0.297 \\
\hline Infection & $5(4.7 \%)$ & $1(0.7 \%)$ & 0.037 \\
\hline Cranial nerve dysfunction & $0(0 \%)$ & $2(1.3 \%)$ & 0.229 \\
\hline Postop CSF leak requiring reop & $2(1.9 \%)$ & $2(1.3 \%)$ & 0.737 \\
\hline Hyponatremia & $24(22.4 \%)$ & $27(18.4 \%)$ & 0.425 \\
\hline 30-day readmission & $6(5.6 \%)$ & $9(6.0 \%)$ & 0.884 \\
\hline Resection & & & 0.303 \\
\hline GTR & $57(53.3 \%)$ & $89(59.7 \%)$ & \\
\hline STR & $50(46.7 \%)$ & $60(40.3 \%)$ & \\
\hline Residual tumor radiographic parameters, cm (range) & & & \\
\hline AP & $1.13(0.3-3.8)$ & $1.48(0.3-3.1)$ & 0.075 \\
\hline ML & $1.18(0.3-4.3)$ & $1.32(0.4-2.9)$ & 0.510 \\
\hline SI & $1.41(1.0-4.0)$ & $1.26(1.0-2.0)$ & 0.564 \\
\hline Diameter & $1.27(0.3-4.3)$ & $1.38(0.7-2.3)$ & 0.617 \\
\hline EVD extera vention
\end{tabular}

EVD $=$ external ventricular drain

* Not all information was available in every patient's medical record on retrospective review. For hyponatremia in the SGA group there were data for 147 patients. For the subcategories of radiographic parameters for residual tumors, in the NCA group there were data for AP in 29 patients, for ML in 24, for SI in 18, and for diameter in 20. In the SGA group there were data for AP in 26 patients, for ML in 24 , for SI in 18, and for diameter in 18.

ly different between the groups. Cavernous sinus invasion was higher in NCAs (43.0\% vs $36.9 \%$ ), although this was not significant. Immunopositivity for p53, a marker of p53 pathway alterations, ${ }^{9}$ was significantly lower in NCAs than in SGAs $(53.1 \%$ vs $72.1 \%, \mathrm{p}=0.041)($ Table 1$)$.

\section{Comparison of Perioperative Complications and Short- Term Outcomes}

We also compared perioperative and short-term outcomes between NCAs, SGAs, and other adenomas. There were no significant differences in length of stay, overall perioperative complications, perioperative use of a lumbar drain, intraoperative CSF encounter, postoperative cranial nerve dysfunction, postoperative CSF leak, or postoperative hyponatremia between the groups (Table 2). Incidence of infection was significantly lower in the SGA group relative to NCAs $(0.7 \%$ vs $4.7 \%, p=0.037)$, although infection was rare in all groups (Table 2). Of the patients with NCA who had postoperative infection, 3 were diagnosed with sinusitis, 1 with meningitis, and 1 with pneumonia. The single infection in the SGA group was an incidence of postoperative sepsis. Readmission rates were also similar between the groups. Although NCAs trended toward a lower GTR rate (53.3\%) when compared to SGAs (59.7\%), there was no significant difference between the groups ( $\mathrm{p}$ $=0.303$ ). There were also no significant differences in the measurements of residual tumor left in patients with STRs (Table 2).

\section{Comparison of Long-Term Outcomes}

We next compared long-term outcomes between our adenoma subgroups (Table 3). NCAs were more likely to receive adjuvant radiation (23.4\%) when compared to SGAs (9.4\%, p = 0.002) (Table 3, Fig. 1), with most of this seemingly driven by a higher incidence of adjuvant radiation therapy, rather than radiosurgery $(12.1 \%$ vs $3.4 \%$, $\mathrm{p}$ $=0.007$ ) $($ Table 3$)$. There was no significant difference between NCAs and SGAs in the incidence of adjuvant radiation provided for residual tumor following resection $(15.0 \%$ vs $7.4 \%, \mathrm{p}=0.052)$. However, NCAs received significantly more adjuvant radiation for recurrence than SGAs $(8.4 \%$ vs $2.0 \%, p=0.017$ ) (Table 3 ). Indeed, NCAs were significantly more likely to recur than SGAs $(9.3 \%$ vs $2.7 \%, \mathrm{p}=0.021$ ). To further explore this, we then generated a Kaplan-Meier plot for progression-free survival for the 2 groups (Fig. 2). The Kaplan-Meier plot demonstrated a significant difference on log-rank analysis $\left(\chi^{2}=4.73, p\right.$ $=0.030$ ). A subsequent backward likelihood ratio Cox regression analysis also demonstrated significantly higher recurrence in NCA tumors versus SGAs (HR 3.64, 95\% CI 1.07-12.30; $p=0.038)$. Other variables that were significantly independently associated with recurrence included 
TABLE 3. Long-term outcomes in 1166 patients with pituitary adenomas

\begin{tabular}{cccc}
\hline \multicolumn{1}{c}{ Variable } & NCA & SGA & p Value \\
\hline Any adjuvant radiation & $25(23.4 \%)$ & $14(9.4 \%)$ & 0.002 \\
\hline Radiosurgery & $12(11.2 \%)$ & $9(6.0 \%)$ & 0.137 \\
\hline Radiotherapy & $13(12.1 \%)$ & $5(3.4 \%)$ & 0.007 \\
\hline Indication: recurrence & $9(8.4 \%)$ & $3(2.0 \%)$ & 0.017 \\
\hline Indication: residual tumor & $16(15.0 \%)$ & $11(7.4 \%)$ & 0.052 \\
\hline Tumor recurrence & $10(9.3 \%)$ & $4(2.7 \%)$ & 0.021 \\
\hline Time to recurrence, mos (range) & $15.9(5-41)$ & $13.5(6-25)$ & 0.705 \\
\hline New postop diabetes insipidus & $13(12.1 \%)$ & $7(4.7 \%)$ & 0.028 \\
\hline Future reop & $5(4.7 \%)$ & $7(4.7 \%)$ & 0.993 \\
\hline Death & $1(0.9 \%)$ & $2(1.3 \%)$ & 0.765 \\
\hline
\end{tabular}

cavernous sinus invasion (HR 3.97, 95\% CI 1.04-15.14; p $=0.043)$ and AP dimension of the tumor (HR 2.23, 95\% CI 1.09-4.59; $\mathrm{p}=0.030$ ) (Table 4).

Compared to NCAs, SGAs had a significantly reduced incidence of new-onset postoperative diabetes insipidus (4.7\% vs $12.1 \%, p=0.028)$ (Fig. 1). Using a binary logistic regression model to evaluate the impact of SGA versus NCA on new diabetes insipidus, NCA was independently associated with increased odds of new postoperative diabetes insipidus (OR 2.39, 95\% CI 0.892-6.42; $p=0.083$ ), although this was not statistically significant. Intraoperative CSF leakage (OR 2.77, 95\% CI 1.03-7.43; $\mathrm{p}=0.043$ ) and tumor cavernous sinus invasion (OR 2.83, 95\% CI $1.05-7.58 ; \mathrm{p}=0.039$ ) were both significantly independently associated with incidence of diabetes insipidus (Table 5). There were no significant differences in the need for future reoperations or death when comparing NCAs and SGAs (Table 3).

\section{Discussion}

Our study includes the largest cohort of patients with SGAs and NCAs with detailed patient characteristics and clinical outcomes from a single institution. SGAs are the most common SPAs, and it is critical to gain a more detailed understanding of them in order to provide optimal care to patients..$^{16}$ Building on a study showing higher MIB-1 staining and a positive correlation between pre- and postoperative TVDTs in NCAs, ${ }^{2}$ in a 2 - to 3 -fold larger cohort, we found that NCAs had a higher incidence of postoperative radiation therapy as well as a higher risk of recurrence and diabetes insipidus relative to SGAs.

The mean ages of patients with NCAs and SGAs were 58 and 59 years old, respectively (Table 1), similar to what has previously been described in the literature ${ }^{2,10}$ and also supporting the literature suggesting that NFPAs present at a later age given the lack of hormonal excess and subsequent symptoms. ${ }^{17} \mathrm{NCAs}$ were relatively evenly distributed between male and female patients, whereas the majority of patients with an SGA were male, similar to multiple reports in the literature ${ }^{10,19}$-although differing with Balogun et al., ${ }^{2}$ who described a female predominance in their study. As expected, the majority of SGAs and NCAs were macroadenomas (Table 1), similar to what has previ- ously been described in the literature. ${ }^{2,5}$ In contrast to the descriptions of Balogun et al., ${ }^{2}$ there was no statistically significant difference between the MIB-1 index of SGAs and NCAs. This discrepancy may be related to the lack of consistent MIB-1 staining in both NCAs and SGAs, with 64/105 and 101/149, respectively, receiving MIB-1 staining. Previous studies have also failed to demonstrate a significant difference in MIB-1 staining between SGAs and NCAs, and the cutoff for MIB-1 remains variable, without a standard provided in the most recent WHO guidelines. ${ }^{22,23}$ Immunopositivity for p53, a marker of TP53 mutations, was significantly higher in SGAs than in NCAs.

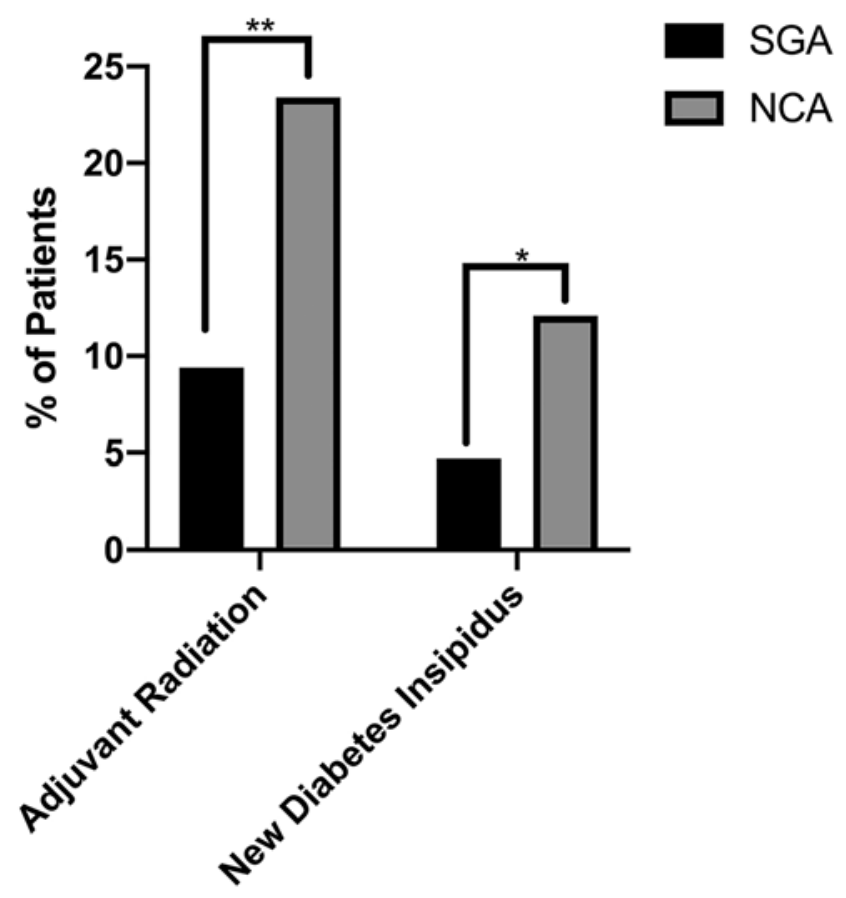

FIG. 1. Incidence of adjuvant radiation and new postoperative diabetes insipidus in NCAs versus SGAs. Patients with NCAs are more likely to develop new postoperative diabetes insipidus than patients with SGAs $\left(4.7 \%\right.$ vs $12.1 \%$, $\left.{ }^{*} p=0.028\right)$. Patients with NCAs are also more likely to require adjuvant radiation therapy $\left(23.4 \%\right.$ vs $\left.9.4 \%,{ }^{* *} p=0.002\right)$. 


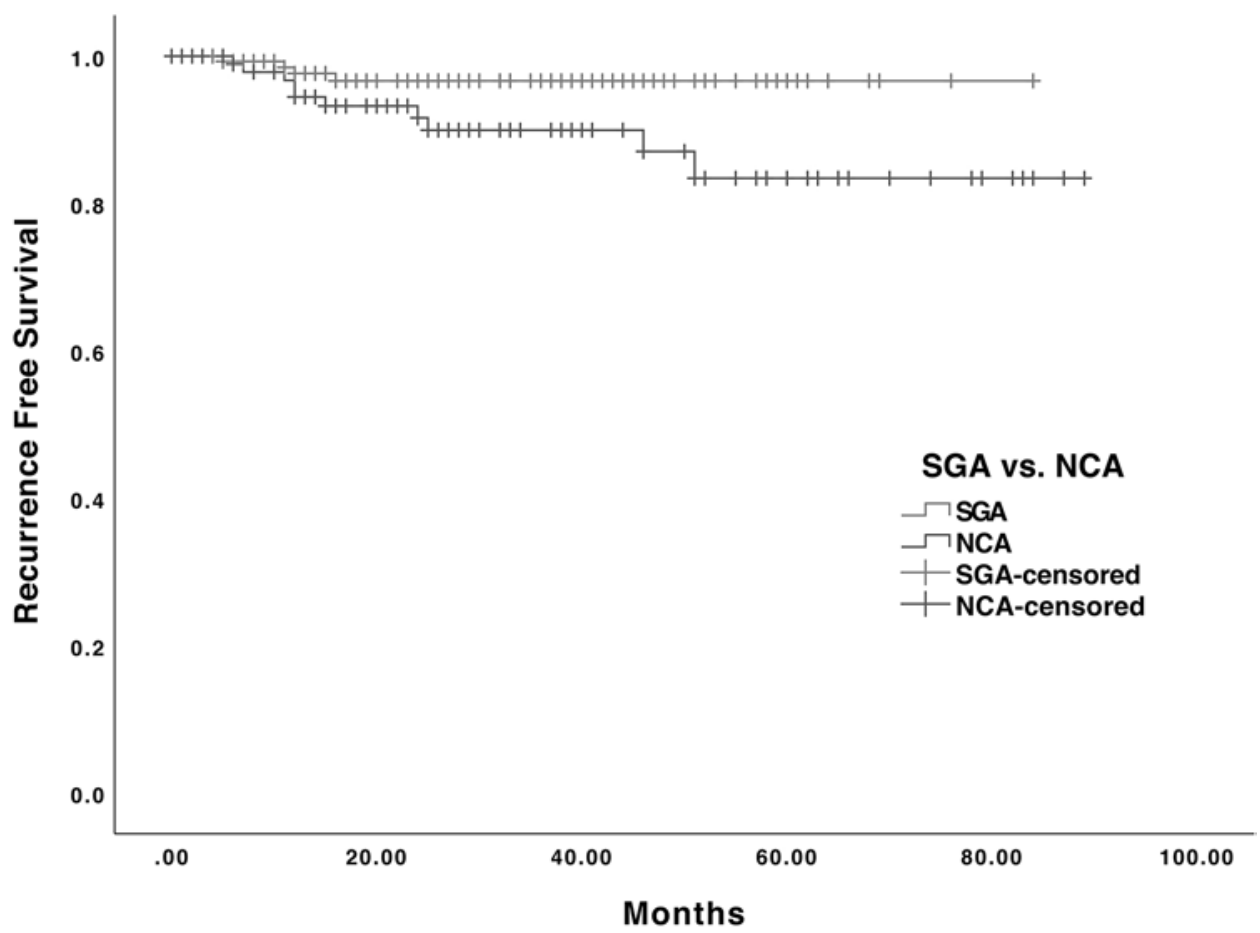

FIG. 2. Kaplan-Meier curve of recurrence-free survival in NCAs versus SGAs. The Kaplan-Meier curve of recurrence-free survival in the SGA and NCA patient population demonstrates reduced recurrence-free survival in patients with NCAs on log-rank analysis $\left(X^{2}=4.73, p=0.030\right)$.

This finding is in agreement with the higher 27 positivity in NCAs reported by Balogun et al. ${ }^{2}$ Both p53 and MIB1 staining theoretically indicate a higher risk category of tumor. ${ }^{3,9,18}$ As a result, we would expect NCAs to have a higher incidence of $\mathrm{p} 53$ staining relative to SGAs, given their more aggressive nature. ${ }^{12}$ Previous studies, however, albeit with limited sample sizes, have demonstrated higher p53 staining in nonfunctioning gonadotroph adenomas relative to NCAs, so our findings are not without precedent. ${ }^{19}$ Future investigation into the relationship between p53 and p27 positivity is required, potentially involving staining of the same SGA and NCA samples for both p27 and p53 to establish inter-test reliability of the 2 stains in these adenomas. Similar to Balogun et al., ${ }^{2}$ we found no significant difference in cavernous sinus invasion between NCAs and SGAs, although there was a trend toward higher invasion in NCAs. We also assessed sphenoid sinus invasion and similarly found no significant differences between the

TABLE 4. Cox regression of variables associated with tumor recurrence

\begin{tabular}{lcc}
\hline \multicolumn{1}{c}{ Variable } & $\mathrm{HR}(95 \% \mathrm{Cl})$ & $\mathrm{p} \mathrm{Value}$ \\
\hline NCA pathology & $3.64(1.07-12.30)$ & 0.038 \\
\hline Cavernous sinus invasion & $3.97(1.04-15.14)$ & 0.043 \\
\hline AP dimension, $\mathrm{cm}$ (range) & $2.23(1.09-4.59)$ & 0.030 \\
\hline ML dimension, $\mathrm{cm}$ (range) & $0.341(0.11-1.02)$ & 0.056 \\
\hline SI dimension, cm (range) & $2.03(0.95-4.35)$ & 0.066 \\
\hline
\end{tabular}

groups (Table 1). Rates of neoadjuvant radiation were low in both groups (Table 1), suggesting that surgeons believed resection alone to be clinically appropriate in most cases.

There were no significant differences between the groups in perioperative outcomes, aside from infection rate (Table 2). SGAs had a significantly lower rate of postoperative infection relative to NCAs, including no incidences of meningitis or sinusitis, although this finding is likely limited by the low overall incidence of postoperative infection. To determine the validity of this finding, further investigation with a larger population of patients would be required. There was no difference in GTR rates (Table 2) between NCAs and SGAs.

Long-term outcomes between the groups were also similar, although with some significant differences. Adjuvant radiation therapy following resection was significantly different between the groups, with SGAs having a rate of $9.4 \%$, but with NCAs having a significantly higher rate of $23.4 \%$ (Table 3, Fig. 1). Adjuvant radiation therapy is often considered for nonfunctioning adenomas in the

TABLE 5. Binary logistic regression of variables associated with new postoperative diabetes insipidus

\begin{tabular}{llc}
\hline \multicolumn{1}{c}{ Variable } & \multicolumn{1}{c}{ OR $(95 \%$ Cl $)$} & p Value \\
\hline Intraop CSF leak & $2.77(1.03-7.43)$ & 0.043 \\
\hline NCA pathology & $2.39(0.892-6.42)$ & 0.083 \\
\hline Cavernous sinus invasion & $2.83(1.05-7.58)$ & 0.039 \\
\hline
\end{tabular}


setting of STR or potentially patient preference, with good outcomes. ${ }^{7,12}$ NCAs were also significantly more likely to recur, which was likely to have contributed to their higher rates of adjuvant radiation therapy (Table 3). When independent predictors of recurrence were assessed using a Cox regression model in patients with either an NCA or an SGA, tumor cavernous sinus invasion, NCA pathology, and AP dimension of the tumor remained independently associated with incidence of recurrence (Table 4). To our knowledge, this represents the first time that NCA pathology has been independently associated with increased risk of recurrence when compared to SGAs.

In agreement with our findings, Balogun et al. ${ }^{2}$ found that NCAs' pre- and postoperative TVDTs were positively associated, in contrast to SGAs, indicating that NCAs that grew quickly prior to surgery continued to grow at a high rate following surgery. They also found that cavernous sinus invasion and low p27 staining were predictors of NCA tumor growth. ${ }^{12}$ Recurrence seems to be impacted by these variables as well; the NCAs in our study had a higher incidence of cavernous sinus invasion, although significantly lower p53 staining. Cavernous sinus invasion was also independently associated with recurrence (Table 4). Further studies could include serial TVDTs to assess any differences in NCAs versus SGAs. Given the relatively low rates of recurrence, future studies with additional patients may also be beneficial. There was no difference in reoperation between the groups (Table 3), suggesting that although NCAs had higher rates of recurrence and adjuvant radiation therapy, those requiring radiation were adequately controlled by the therapy.

SGAs also had significantly lower levels of diabetes insipidus relative to the NCAs (Table 3). Risk factors for postoperative diabetes insipidus from pituitary surgery include the following: visual abnormalities, suprasellar extension of the tumor, intraoperative CSF leakage, and maximal tumor diameter. ${ }^{15,20}$ On multivariate analysis using binary logistic regression modeling, NCA pathology was independently associated with increased incidence of new diabetes insipidus, although this only trended toward significance $(\mathrm{p}=0.083)$. Corresponding with what has previously been described in the literature,,$^{15,20}$ intraoperative CSF leakage was independently and significantly associated with diabetes insipidus. Cavernous sinus invasion was also significantly associated with new postoperative diabetes insipidus on multivariate analysis. We suspect that this may be a result of the additional dissection required to remove these tumors, potentially increasing the risk of disturbing the pituitary stalk. Additional studies with larger patient sample sizes are required to fully elucidate the association between cavernous sinus invasion and postoperative diabetes insipidus. Because our study only included NFPAs, studies involving functional adenomas as well should be performed to determine if the association between cavernous sinus invasion and diabetes insipidus is NFPA-specific. Further investigation into differences in new postoperative diabetes insipidus between NCAs and SGAs is also required, given the small incidence of diabetes insipidus in our study, to determine if patients with NCAs are at higher risk for postoperative diabetes insipidus relative to SGAs.
Although the behavioral characteristics of SGAs and NCAs have been compared in the past, ${ }^{12}$ there remains a paucity of data comparing the clinical outcomes between the 2 groups. Our study, to our knowledge, is the largest cohort of SGAs and NCAs with detailed patient characteristics as well as short- and long-term clinical outcomes, including diabetes insipidus, the need for adjuvant radiation therapy, time to recurrence, and incidence of reoperation, with an average follow-up of more than 2 years for all subgroups. We found that patients with NCAs have a higher incidence of postoperative diabetes insipidus and are at higher risk for recurrence relative to patients with SGAs.

Limitations of our study include its retrospective nature, lack of tumor volumetric data, and the relatively low number of patients in each group, albeit that limitation was due to the rarity of the pathological entities. Future directions could involve including tumor volumetric data and larger numbers of patients, perhaps using a multiinstitutional database, to provide further insight into the differences between NCAs and SGAs. As transcription factor staining for SF-1, PIT-1, and TPIT becomes more widespread, additional studies could expand on this study by using the full repertoire of transcription factor staining when determining SGA and NCA subgroups. Follow-up studies could also investigate underlying biological mechanisms differentiating these 2 groups and could leverage techniques such as regional biopsies and single-cell RNA sequencing to investigate whether there is heterogeneity at various levels within these tumors.

\section{Conclusions}

Our study highlights differences in patient characteristics and clinical outcomes between NCAs and SGAs, supporting the concept that they should be considered distinct groups, as recognized in the 2017 WHO guidelines. SGAs have a much higher incidence in male patients. Relative to SGAs, NCAs have increased risk of recurrence and postoperative diabetes insipidus, both of which could reflect more biologically aggressive tumors. The rarity of these 2 subgroups limits the potential sample size of any study, highlighting the need for further multi-institutional studies to support our findings. Future studies investigating the underlying biological differences between NCAs and SGAs should also be considered.

\section{Acknowledgments}

We thank the UCSF Brain Tumor Research Center for its continued support.

\section{References}

1. Asa SL, Mete O: What's new in pituitary pathology? Histopathology 72:133-141, 2018

2. Balogun JA, Monsalves E, Juraschka K, Parvez K, Kucharczyk W, Mete O, et al: Null cell adenomas of the pituitary gland: an institutional review of their clinical imaging and behavioral characteristics. Endocr Pathol 26:63-70, 2015

3. Chiloiro S, Bianchi A, Doglietto F, de Waure C, Giampietro A, Fusco A, et al: Radically resected pituitary adenomas: prognostic role of $\mathrm{Ki} 67$ labeling index in a monocentric retrospective series and literature review. Pituitary 17:267-276, 2014 
4. Daly AF, Rixhon M, Adam C, Dempegioti A, Tichomirowa MA, Beckers A: High prevalence of pituitary adenomas: a cross-sectional study in the province of Liege, Belgium. J Clin Endocrinol Metab 91:4769-4775, 2006

5. Drange MR, Fram NR, Herman-Bonert V, Melmed S: Pituitary tumor registry: a novel clinical resource. J Clin Endocrinol Metab 85:168-174, 2000

6. Drummond J, Roncaroli F, Grossman AB, Korbonits M: Clinical and pathological aspects of silent pituitary adenomas. J Clin Endocrinol Metab 104:2473-2489, 2019

7. Erridge SC, Conkey DS, Stockton D, Strachan MWJ, Statham PFX, Whittle IR, et al: Radiotherapy for pituitary adenomas: long-term efficacy and toxicity. Radiother Oncol 93:597-601, 2009

8. Gittleman H, Ostrom QT, Farah PD, Ondracek A, Chen Y, Wolinsky Y, et al: Descriptive epidemiology of pituitary tumors in the United States, 2004-2009. J Neurosurg 121:527-535, 2014

9. Hasanov R, Aydoğan Bİ, Kiremitçi S, Erden E, Güllü S: The prognostic roles of the Ki-67 proliferation index, P53 expression, mitotic index, and radiological tumor invasion in pituitary adenomas. Endocr Pathol 30:49-55, 2019

10. Ho DMT, Hsu CY, Ting LT, Chiang H: The clinicopathological characteristics of gonadotroph cell adenoma: a study of 118 cases. Hum Pathol 28:905-911, 1997

11. Ioachimescu AG, Eiland L, Chhabra VS, Mastrogianakis GM, Schniederjan MJ, Brat D, et al: Silent corticotroph adenomas: Emory University cohort and comparison with ACTH-negative nonfunctioning pituitary adenomas. Neurosurgery 71:296-304, 2012

12. Loeffler JS, Shih HA: Radiation therapy in the management of pituitary adenomas. J Clin Endocrinol Metab 96:19922003, 2011

13. Mayson SE, Snyder PJ: Silent (clinically nonfunctioning) pituitary adenomas. J Neurooncol 117:429-436, 2014

14. Mete O, Lopes MB: Overview of the 2017 WHO Classification of Pituitary Tumors. Endocr Pathol 28:228-243, 2017

15. Nayak P, Montaser AS, Hu J, Prevedello DM, Kirschner LS, Ghalib L: Predictors of postoperative diabetes insipidus following endoscopic resection of pituitary adenomas. J Endocr Soc 2:1010-1019, 2018

16. Nishioka H, Inoshita N, Mete O, Asa SL, Hayashi K, Takeshita A, et al: The complementary role of transcription factors in the accurate diagnosis of clinically nonfunctioning pituitary adenomas. Endocr Pathol 26:349-355, 2015

17. Ntali G, Wass JA: Epidemiology, clinical presentation and diagnosis of non-functioning pituitary adenomas. Pituitary 21:111-118, 2018

18. Ramírez C, Cheng S, Vargas G, Asa SL, Ezzat S, González B, et al: Expression of Ki-67, PTTG1, FGFR4, and SSTR 2, 3, and 5 in nonfunctioning pituitary adenomas: a high throughput TMA, immunohistochemical study. J Clin Endocrinol Metab 97:1745-1751, 2012
19. Scheithauer BW, Gaffey TA, Lloyd RV, Sebo TJ, Kovacs KT, Horvath E, et al: Pathobiology of pituitary adenomas and carcinomas. Neurosurgery 59:341-353, 2006

20. Schreckinger M, Szerlip N, Mittal S: Diabetes insipidus following resection of pituitary tumors. Clin Neurol Neurosurg 115:121-126, 2013

21. Tjörnstrand A, Gunnarsson K, Evert M, Holmberg E, Ragnarsson $\mathrm{O}$, Rosén $\mathrm{T}$, et al: The incidence rate of pituitary adenomas in western Sweden for the period 2001-2011. Eur J Endocrinol 171:519-526, 2014

22. Wolfsberger S, Wunderer J, Zachenhofer I, Czech T, BöcherSchwarz HG, Hainfellner J, et al. Expression of cell proliferation markers in pituitary adenomas - correlation and clinical relevance of MIB-1 and anti-topoisomerase-II $\alpha$. Acta Neurochir (Wien) 146:831-839, 2004

23. Zhao D, Tomono Y, Nose T: Expression of P27 $7^{\mathrm{kipl}}$ and Ki-67 in pituitary adenomas: an investigation of marker of adenoma invasiveness. Acta Neurochir (Wien) 141:187-192, 1999

\section{Disclosures}

The authors report no conflict of interest concerning the materials or methods used in this study or the findings specified in this paper.

\section{Author Contributions}

Conception and design: Aghi, Haddad, Young, MP Pereira, El-Sayed. Acquisition of data: Haddad, MP Pereira, Joshi, KM Pereira, Osorio, Donohue, Peeran, Sudhir, Jain, Beniwal, Chopra, Sandhu, Theodosopoulos, Kunwar, Gurrola, Blevins. Analysis and interpretation of data: Haddad, MP Pereira. Drafting the article: Haddad, Young, Oh, Joshi. Critically revising the article: Aghi, Haddad, Oh, MP Pereira, Joshi. Reviewed submitted version of manuscript: Aghi, Haddad, Oh, Theodosopoulos, Kunwar, El-Sayed, Gurrola, Blevins. Statistical analysis: Haddad, MP Pereira. Administrative/technical/material support: Haddad, Theodosopoulos, Kunwar, El-Sayed, Gurrola, Blevins. Study supervision: Aghi, Haddad.

\section{Correspondence}

Manish K. Aghi: University of California, San Francisco, CA. manish.aghi@ucsf.edu. 